\title{
Quasi-local conformal Killing horizons: Classical phase space and the first law
}

\author{
Ayan Chatterjee* \\ Department of Physics and Astronomical Science, \\ Central University of Himachal Pradesh, Dharamshala -176215, India. \\ Avirup Ghosh ${ }^{\dagger}$ \\ Theory Division, Saha Institute of Nuclear Physics, 1/AF Bidhan Nagar, Kolkata 700064, INDIA.
}

\begin{abstract}
In realistic situations, black hole spacetimes do not admit a global timlike Killing vector field. However, it is possible to describe the horizon in a quasi-local setting by introducing the notion of a quasi-local boundary with certain properties which mimic the properties of a black hole inner boundary. Isolated horzons and Killing horizons are examples of such kind. In this paper, we construct such a boundary of spacetime which is null and admits a conformal Killing vector field. Furthermore we construct the space of solutions (in general relativity) which admits such quasi-local conformal Killing boundaries. We also establish a form of first law for these quasi-local horizons.
\end{abstract}

PACS numbers: 04.70.Dy, 04.60.-m, 04.62.+v

* ayan.theory@gmail.com

$\dagger$ avirup.ghosh@saha.ac.in 


\section{INTRODUCTION}

A black hole is described to be a region of spacetime where the gravitational attraction is high enough to prevent even light from escaping to infinity. In asymptotically flat spacetimes, the impossibility of light escaping to future null infinity form the appropriate characterization of a black hole. In other words, this region lies outside the causal past of the future null infinity $\mathscr{I}^{+}$. The boundary of such a region is called the event horizon $\mathscr{H}[1,2]$. To be more precise, consider a strongly asymptotically predictable spacetime $\left(\mathcal{M}, g_{a b}\right)$. The spacetime is said to contain a black hole if $\mathcal{M}$ is not contained in $J^{-}\left(\mathscr{I}^{+}\right)$. The black hole region is denoted by $\mathscr{B}=\mathcal{M}-J^{-}\left(\mathscr{I}^{+}\right)$and the event horizon is the boundary of $\mathscr{B}$ (alternatively it may also be defined as the future boundary of past of future null infinity: $\left.\mathscr{H}=\partial\left[J^{-}\left(\mathscr{I}^{+}\right)\right]\right)$. The definition of event horizon thus requires that we are able to construct the future null infinity $\mathscr{I}^{+}$. This implies that the entire future of the spacetime needs to be known beforehand to ensure the existence of an event horizon. Indeed, the condition of strong asymptotic predictibility of spacetime signifies that we have a complete knowledge of the future evolution. From the above consideration, it is clear that $\mathscr{H}$ is a global concept and it becomes difficult to proceed much further using this definition. However, the notions simplify for stationary spacetimes which are expected states of black holes in equilibrium. In equilibrium, these spacetimes admit Killing symmetries and thus exhibit a variety of interesting features. Indeed, the strong rigidity theorem implies that the event horizon of a stationary black hole is a Killing horizon [3]. However not all Killing horizons are event horizons. Killing horizons only require a timelike Killing vector field in the neighbourhood of the horizon whereas construction of a stationary event horizon requires a global timelike Killing vector field.

The identification of the event horizon of a stationary black hole to a Killing horizon was useful to prove the laws of mechanics for event horizons [4]. It was shown that in general relativity, the surface gravity $\kappa_{H}$ of a stationary black hole must be a constant over the event horizon. The first law of black hole mechanics refers to stationary space-times admitting an event horizon and small perturbations about them. This law states that the differences in mass $M$, area $A$ and angular momentum $J$ to two nearby stationary black hole solutions are related through $\delta M=\kappa_{H} \delta A / 8 \pi+\Omega_{H} \delta J$. One gets additional terms like charge if matter fields are present. Hawking's proof that due to quantum particle creation, black holes radiate to infinity, particles of all species at a temperature $\kappa_{H} / 2 \pi$, implied that laws of black hole mechanics are the laws of thermodynamics of black holes [5]. Moreover, the entropy of the black holes must be proportional to it's area [6, 7].

However, it was realised very soon that this identification of entropy to area leads to new difficulties. Classical general relativity gives rise to infinite number of degrees of freedom but it is not clear if the laws of thermodynamics can arise out of a statistical mechanical treatment of these classical information (see [8]). One must find ways to extract quantum degrees of freedom of general relativity. The framework of Killing Horizon was broadened to understand the origin of entropy and black hole thermodynamics [9-15]. It turned out that the framework of Isolated Horizons (IH) was more suited to address these questions from the perspective of loop quantum gravity [16-22]. It is argued that the effective quantum degrees of freedom which capture the thermodynamic information of black holes are localised, more precisely, reside on the horizon. Isolated horizons are suited for this description since they capture only the local information; isolated horizons are local descriptions of horizons and unlike event horizons, do not require the global history of spacetime [23-31]. It arises that the effective field theory induced on a IH is a Chern- Simons theory whose quantisation and counting of states is consistent with the results of Bekenstein and Hawking. Moreover, since IH replaced the global notion of event horizons with a local description, the requirement of a knowledge of full space-time history as well as the asymptotics is avoided (see [32, 33] for a first order description of theories with topological terms). The underlying spacetime therefore might not admit a global Killing vector at all in the isolated framework. While this has been a significant development in the understanding of black hole mechanics, generalizations to dynamically evolving horizons has also been reported [36-38]. These dynamical horizons are closely related to the notion of trapping horizons developed earlier [34, 35]. Using the boundary conditions for dynamical horizon it was shown that a flux balance law, relating the change of area of the dynamical horizon to the flux of the matter energy, exists, reproducing an integrated version of a first law [36-38]. Moreoever, it has also been shown that if the horizon is slowly evolving, a form of the first law arises [39-41]. The construction of a phase space for these horizons has also been carried out in the metric variables.

Another class of horizons that has been of interest are conformal Killing horizons (CKH). Though not a trapping horizon it essentially captures a dynamical situation. The notion of CKH and it's properties were developed in [42-46]. These are null hypersurfaces whose null geodesics are orbits of a conformal Killing field. If $\xi^{a}$ is a vector field which satisfies $£_{\xi} g_{a b}=2 f g_{a b}$, and is null, it generates a $\mathrm{CKH}$ for the metric $g_{a b}$. It has been shown that an analogue of the zeroth law holds for a conformal Killing horizon as well. More precisely, since $\xi^{a}$ generates a null surface, it is geodesic and one can define an accelration through $\xi^{b} \nabla_{b} \xi^{a}=\kappa_{\xi} \xi^{a}$. Then, the quantity $\left(\kappa_{\xi}-2 f\right)$ which essentially is a combination of the acceleration of the conformal Killing vector and the conformal factor, can be shown to be Lie dragged along the horizon and can therefore be interpreted as a temperature. An analogue of the first law is therefore expected to hold in this case as well but has not been established in the literature. In this paper, we address the 
question if a form of the first law can be established at all for a CKH. As we discuss below, if such a law exists, it may lead to some important clues for a dynamically evolving horizon.

The plan of the paper is as follows. We start by developing the geometry of a quasi-local conformal Killing horizon. We assume that a spacetime time region $\mathcal{M}$ has a null boundary $\Delta$ which however may have non- zero expansion $(\theta=-2 \rho \neq 0)$. In other words we take the null generators of $\Delta$ to be only shear-free. We observe that these conditions are enough to ensure that the null generators $l^{a}$ are conformal Killing vectors on $\Delta$. Now, since these null surfaces are not expansion free, they may be growing; in fact $£_{l}{ }^{2} \epsilon=\theta^{2} \epsilon$ and hence, are good candidates for growing horizons. The situation in some sense mimics what one has at null infinity in an asymptotically flat space-time. However, we are more interested in an inner horizon. The physical situation for these horizons can be visualised as follows. Suppose matter falls in through a horizon as a result of which it grows (supposing that matter satisfies standard energy conditions) and hence has a non- zero positive expansion. When this matter flux stops to fall in through the horizon, by the Raychaudhuri equation, an initially positively expanding horizon will slow down it's expansion and after some time reach the state of equilibrium. This equilibrium state has zero expansion and it's geometrical set- up has been developed through the Isolated horizon formulation. We are interested to construct the space of solutions of only those dynamically evolving horizons which can be generated by a conformal Killing vector field. By construction, the CKH admit a limit to the IH formulation. We suppose that the matter flux across $\Delta$ be a real scalar field $(\varphi)$ satisfying the condition $£_{l} \varphi=-2 \rho \varphi$ on the horizon. The geometrical conditions ensures that a form of zeroth law exists. In the next section, we show that the action for general relativity admits a well defined variational principle in presence of the conformal Killing horizon boundary and proceed to construct the symplectic structure. An interesting outcome is the construction of the phase space, identification of a boundary symplectic structure and the existence of a first law. Further, it arises that gravity and matter together gives a well defined phase- space provided a balance condition holds. This balance condition turns out to be nothing but Einstein's equation contracted with the null generators $l^{a}$ (say). We thus get a quasi-local analogue of a conformal Killing horizon.

\section{GEOMETRICAL SETTING AND BOUNDARY CONDITIONS}

In this section, we introduce the minimal set of boundary conditions which are suitable for a quasilocal conformal Killing horizon. We assume that all fields under consideration are smooth. Let $\mathcal{M}$ be a 4 - manifold equipped with a metric $g_{a b}$ of signature $(-,+,+,+)$. Consider a null hypersurface $\Delta$ of $\mathcal{M}$ with $l^{a}$ being it's future directed null normal. Given this null normal $l^{a}$, one can introduce another future directed null vector field $n^{a}$ which is transverse to $\Delta$. Further, one has a set of complex null vector field $(m, \bar{m})$, which are tangential to $\Delta$. This null tetrad $(l, n m \bar{m})$ constitutes the Newman- Penrose basis. The vector fields satisfy the condition that $l . n=-1=-m \cdot \bar{m}$, while all other scalar products vanish. Let $q_{a b}$ be the degenerate metric on the hypersurface. The expansion $\theta_{l}$ of the null normal is given by $q^{a b} \nabla_{a} l_{b}$. In terms of the Newman- Penrose co-effecients, $\theta_{l}=-2 \rho$ (see appendix A and [47] for details). The accelaration of $l^{a}$ follows from the expression $l^{a} \nabla_{a} l_{b}=(\epsilon+\bar{\epsilon}) l_{b}$ and is given by $\kappa_{l}:=\epsilon+\bar{\epsilon}$. To avoid cumbersome notation, we will do away with the subscripts $(l)$ from now on if no confusion arises. It would be useful to define an equivalance class of null normals $\left[l^{a}\right]$ such that two null normals $l$ and $l^{\prime}$ will be said to belong to the same equivalance class if $l^{\prime}=c l$ where $c$ is a constant on $\Delta$.

\section{Quasi-local conformal horizon}

Definition: A null hypersurface $\Delta$ of $\mathcal{M}$ will be called quasi-local conformal horizon if the following conditions hold.:

1. $\Delta$ is topologically $S^{2} \times R$ and null.

2. The shear $\sigma$ of $l$ vanishes on $\Delta$ for any null normal $l$.

3. All equations of motion hold at $\Delta$ and the stress- energy tensor $T_{a b}$ on $\Delta$ is such that $-T^{a}{ }_{b} l^{b}$ is future directed and causal.

4. If $\varphi$ is a matter field then it must satisfy $£_{l} \varphi=-2 \rho \varphi$ on $\Delta$ for all null normals $l$.

5. The quantity $[2 \rho+\epsilon+\bar{\epsilon}]$ is Lie dragged for any null-normal $l$.

Some comments on the boundary conditions are in order. The first condition imposes restrictions on the topology of the hypersurface. It is natural to motivate this condition from Hawking's theorem on the topology of black holes in 
asymptotically flat stationary spacetimes or it's extension [3, 48]. But, we are also interested in spacetimes which are aymptotically non- flat or that they are non- stationary for which, these theorems may not hold true. However it is not unnatural to argue that since black hole horizons forming out of gravitational collapse have spherical topologies, such conditions might exist. This condition is also assumed in the Isolated Horizon formalism. For these isolated hypersurfaces, the expansion $\theta$ of the null normal $l^{a}$ vanishes (which is not true in our case). It is possible that cross- sections of such quasilocal horizons may admit other topologies. For the time being, we would not include such generalities and only retain the condition that the cross- sections of the hypersurfaces are spherical.

The second boundary condition on the shear is a simplification. Shear measures the amount of gravitational flux flowing across the surface, and we put the gravity flux to be vanishing. This boundary condition on the shear $\sigma$ of null normal $l^{a}$ has several consequences. First, since $l_{a}$ is hypersurface orthogonal, the Frobenius theorem implies that $\rho$ is real and $\kappa=0$. Secondly, the Ricci identity can be written as

$$
D \sigma-\delta \kappa=\sigma(\rho+\bar{\rho}+3 \epsilon-\bar{\epsilon})-\kappa(\tau-\bar{\pi}+\bar{\alpha}+3 \beta)+\Psi_{0},
$$

where $D=l^{a} \nabla_{a}, \delta=m^{a} \nabla_{a}, \Psi_{0}$ is one of the Weyl scalars and the other quantities are the Newman- Penrose scalars (see [47] for details). If $\sigma \triangleq 0$, it implies $\Psi_{0} \triangleq 0$. Next, since $l^{a}$ is null normal to $\Delta$, it is twist- free and a geodetic vector field. The implications of $l^{a}$ being twist- free has already been shown above. The accelaration of $l^{a}$ follows from the expression $l^{a} \nabla_{a} l^{b}=(\epsilon+\bar{\epsilon}) l^{b}$ and is given by $\kappa_{l}:=\epsilon+\bar{\epsilon}$. The acceleration of the null normal varies over the equivalence class $[c l]$ where $c$ is a constant on $\Delta$. This is only natural that the acceleration varies in the class since in the absence of the knowledge of asymptotics, the acceleration cannot be fixed.

Further, it can be seen that the null normal $l^{a}$ is such that

$$
\underset{\nabla_{(a} l_{b)}}{\triangleq}-2 \rho m_{(a} \bar{m}_{b)}
$$

which implies that $l^{a}$ is a conformal Killing vector on $\Delta$. Moreover, the Raychaudhuri equation implies that $R_{a b} l^{a} l^{b} \neq 0$ and hence $-R_{b}^{a} l^{b}$ can have components which are tangential as well as transverse to $\Delta$.

The third boundary condition only implies that the field equations of gravity be satisfied and that the matter fields be such that their energy momentum tensor satisfies some mild energy conditions. The fourth and the fifth boundary conditions are somewhat adhoc but can be motivated. Let us first look at the fourth boundary condition. We have kept open the possibility that matter fields may cross the horizon and the horizon may grow. The matter field is taken to be a massless scalar field $\varphi$ which behaves in a certain way which mimics it's conformal nature. The fifth condition is motivated by the fact that surface gravity remains invariant under conformal transformations [44, 45]. It can be shown that the quantity that is constant for these horizons is $(2 \rho+\epsilon+\bar{\epsilon})$. A conformal transformation of the metric amounts to a conformal transformation of the two-metric on $\Delta$. Under a conformal transformation $g_{a b} \rightarrow \Omega^{2} g_{a b}$ and one needs a new covariant derivative operator which annihilates the conformally transformed metric. Under such a conformal transformation $l^{a} \rightarrow l^{a}, l_{a} \rightarrow \Omega^{2} l_{a}, n^{a} \rightarrow \Omega^{-2} n^{a}, n_{a} \rightarrow n_{a}, m^{a} \rightarrow \Omega^{-1} m^{a}, m_{a} \rightarrow \Omega m_{a}$. The new derivative operator is such that it transforms as

$$
\nabla_{a} l_{b} \rightarrow \Omega^{2} \nabla_{a} l_{b}+2 \Omega \partial_{a} \Omega l_{b}-\Omega^{2}\left[l_{c} \delta_{a}^{c} \partial_{b} \log \Omega+l_{c} \delta_{b}^{c} \partial_{a} \log \Omega-g_{a b} g^{c d} l_{c} \partial_{d} \log \Omega\right]
$$

If one defines a one- form $\omega_{a} \triangleq-n^{b} \nabla_{a} l_{b}$, it transforms under the conformal transformation as

$$
\tilde{\omega}_{a} \triangleq \omega_{a}+2 \partial_{a} \log \Omega-\partial_{a} \log \Omega-n_{a} l^{c} \partial_{c} \log \Omega
$$

It follows that the Newman- Penrose scalars transform in the following way

$$
\begin{aligned}
& \widetilde{(\epsilon+\bar{\epsilon})} \triangleq(\epsilon+\bar{\epsilon})+2 £_{l} \log \Omega \\
& \tilde{\rho} \triangleq \rho-£_{l} \log \Omega \\
& \tilde{\sigma} \triangleq \sigma
\end{aligned}
$$

where, $\rho=-m^{a} \bar{m}^{b} \nabla_{a} l_{b}$ and $\sigma=-\bar{m}^{a} \bar{m}^{b} \nabla_{a} l_{b}$. Thus it follows that $2 \rho+\epsilon+\bar{\epsilon}$ remains invariant under a conformal transformation.

At this point, it would be useful to recall the boundary conditions of a weakly isolated horizon and note the important differences. A weakly isolated horizon is a null hypersurface which satisfies the first and the third boundary conditions given here and that the expansion of the null normal $l^{a}$ be zero. On such surface, there exists a one- form $\omega_{a}$ which is also assumed to be Lie dragged by the vector field $l^{a}$. Thus, instead of the condition on shear, for a WEH, the expansion of the null normal $l^{a}$ is taken to be vanishing, $\theta=0=2 \rho$. By the Raychaudhuri equation, the boundary conditions imply that the shear is zero and that no matter field crosses the horizon (and hence the name 
isolated). However, here, we impose only the condition that the shear vanishes and keep the possibility that matter fields may fall through the surface (but no gravitational flux) and that the hypersurface may grow along the affine parameter. As we shall show, removing our last condition does not restrict one to define a well defined phase space, but is essential to get a first law. It is an analogue of the condition $£_{l}(\epsilon+\bar{\epsilon}) \triangleq 0$ assumed in the case of weakly isolated horizon. It may be useful to note that the fifth boundary condition as given above, can be recast is a form which is an analogue of that for a weakly isolated horizon by setting $£_{l} \tilde{\omega}=0$, where $\tilde{\omega}_{a} \triangleq \omega_{a}+\partial_{a} \log \Omega-n_{a} l^{c} \partial_{c} \log \Omega$ and the conformal factor is set such that $£_{l} \log \Omega=\rho$.

\section{ACTION PRINCIPLE AND THE CLASSICAL PHASE SPACE}

We are interested in constructing the space of solutions of general relativity, and we use the first order formalism in terms of tetrads and connections. This formalism is naturally adapted to the nature of the problem in the sense that the boundary conditions are easier to implement. Moreover it has the advantage that the construction of the covariant phase- space becomes simpler. For the first order theory, we take the fields on the manifold to be $\left(e_{a}^{I}, A_{a I}{ }^{J}, \varphi\right)$, where $e_{a}^{I}$ is the co- tetrad, $A_{a I}{ }^{J}$ is the gravitational connection and $\varphi$ is the scalar field. The Palatini action in first order gravity with a scalar field is given by:

$$
S_{G+M}=-\frac{1}{16 \pi G} \int_{\mathcal{M}}\left(\Sigma^{I J} \wedge F_{I J}\right)-\frac{1}{2} \int_{\mathcal{M}} d \varphi \wedge \star d \varphi
$$

where $\Sigma^{I J}=\frac{1}{2} \epsilon^{I J}{ }_{K L} e^{K} \wedge e^{L}, A_{I J}$ is a Lorentz $S O(3,1)$ connection and $F_{I J}$ is a curvature two-form corresponding to the connection given by $F_{I J}=d A_{I J}+A_{I K} \wedge A^{K}$. The action might have to be supplemented with boundary terms to make the variation well defined.

\section{Variation of the action}

For the variational principle, we consider the spacetime to be bounded by a null surface $\Delta$, two Cauchy surfaces $M_{+}$and $M_{-}$which extend to the asymptotic infinity. The boundary conditions on the fields are the following. At the asymptotic infinity, the fields satisfy appropriate boundary conditions. The fields on the hypersurfaces $M_{+}$and $M_{-}$are fixed so that their variations vanish. On the surface $\Delta$, we fix a set of internal null- tetrad $\left(l^{I}, n^{I}, m^{I}, \bar{m}^{I}\right)$ such that the flat connection annihilates them. The fields on the manifold $\left(e_{a}^{I}, A_{a I}{ }^{J}, \varphi\right)$, must satisfy the following conditions. First, on $\Delta$, the configurations of the tetrads be such that $l^{a}=e_{I}^{a} l^{I}$ are the null vectors which satisfy the boundary conditions for quasi- local conformal horizon. Second, the possible connnections also satisfy the boundary conditions and be such that $(2 \rho+\epsilon+\bar{\epsilon})$ is constant. Thirdly, we consider all those configurations of scalar field which, on $\Delta$, satisfy $£_{l} \varphi=-2 \rho \varphi$.

We now check that the variational principle is well- defined if the boundary conditions on the fields, as given above, hold. However, we need some expressions for tetrads and connections on $\Delta$, details of which are given in the appendix A. On the conformal horizon, the $\Sigma^{I J}$ is given by

$$
\sum^{I J} \triangleq 2 l^{[I} n^{J]}{ }^{2} \epsilon+2 n \wedge\left(i m l^{[I} \bar{m}^{J]}-i \bar{m} l^{[I} m^{J]}\right)
$$

and the connection is given by

$$
\begin{aligned}
\stackrel{A_{a I J}}{\longleftarrow} & \triangleq 2\left[(\epsilon+\bar{\epsilon}) n_{a}-(\bar{\alpha}+\beta) \bar{m}_{a}-(\alpha+\bar{\beta}) m_{a}\right] l_{[I} n_{J]}+2\left(-\bar{\kappa} n_{a}+\bar{\rho} \bar{m}_{a}\right) m_{[I} n_{J]}+2\left(-\kappa n_{a}+\rho m_{a}\right) \bar{m}_{[I} n_{J]} \\
& +2\left(\pi n_{a}+-\mu \bar{m}_{a}-\lambda m_{a}\right) m_{[I} l_{J]}+2\left(\bar{\pi} n_{a}-\bar{\mu} m_{a}-\bar{\lambda} \bar{m}_{a}\right) \bar{m}_{[I} l_{J]} \\
& +2\left[-(\epsilon-\bar{\epsilon}) n_{a}+(\alpha-\bar{\beta}) m_{a}+(\beta-\bar{\alpha}) \bar{m}_{a}\right] m_{[I} \bar{m}_{J]} .
\end{aligned}
$$

The Lagrangian 4- form for the fields $\left(e_{a}{ }^{I}, A_{a I}{ }^{J}, \varphi\right)$ is given in the following way.

$$
L_{G+M}=-\frac{1}{16 \pi G}\left(\Sigma^{I J} \wedge F_{I J}\right)-\frac{1}{2} d \varphi \wedge \star d \varphi .
$$

The first variation of the action leads to equations of motion and boundary terms. The equations of motion consist of the following equations. First, variation of the action with respect to the connection implies that the curvature $F^{I J}$ is related to the Riemann tensor $R^{c d}$, through the relation $F_{a b}{ }^{I J}=R_{a b}{ }^{c d} e_{c}^{I} e_{d}^{J}$. Second, variation with respect to the 
tetrads lead to the Einstein equations and third, the first variation of the matter field gives the equation of motion of the matter field. On- shell, the first variation is given by the following boundary terms

$$
\delta L_{G+M}:=d \Theta(\delta)=-\frac{1}{16 \pi G} d\left(\Sigma^{I J} \wedge \delta A_{I J}\right)-d(\delta \varphi \star d \varphi)
$$

which are to be evaluated on the boundaries $M_{-}, M_{+}$, asymptotic infinity and $\Delta$. However, since fields are set fixed on the initial and the final hypersurfaces they vanish. The boundary conditions at infinity are assumed to be appropriately chosen and they can be suitably taken care of. The only terms which are of relevance for this case are the terms on the internal boundary. On the internal boundary $\Delta$, the boundary terms give (see appendix B for details)

$$
16 \pi G \delta L_{G+M}=-\delta\left(\frac{\mathbf{R}_{11}}{\rho} n\right) \wedge^{2} \epsilon-\delta\left(2 \rho n \wedge^{2} \epsilon\right)+8 \pi G \delta\left(\frac{\mathbf{T}_{11}}{\rho} n\right) \wedge{ }^{2} \epsilon
$$

Since Einstein's equations give $R_{11}=8 \pi G T_{11}$, the first and the third term cancel and only $\left(2 \rho n \wedge{ }^{2} \epsilon\right)$ remains. Thus, if one adds the term $16 \pi G S^{\prime}=\int_{\Delta}\left(2 \rho n \wedge{ }^{2} \epsilon\right)$ to the action, it is well defined for the set of boundary conditions on $\Delta$. As we shall see below, since this is a boundary term, it does not contribute to the symplectic structure.

\section{Covariant phase- space and the symplectic Structure}

For a general Lagrangian, the on-shell variation gives $\delta L=d \Theta(\delta)$ where $\Theta$ is called the symplectic potential. It is a 3 -form in space-time and a 0 -form in phase space. Given the symplectic potential, one can construct the symplectic structure $\Omega\left(\delta_{1}, \delta_{2}\right)$ on the space of solutions. One first constructs the symplectic current $J\left(\delta_{1}, \delta_{2}\right)=\delta_{1} \Theta\left(\delta_{2}\right)-\delta_{2} \Theta\left(\delta_{1}\right)$, which, by definition, is closed on-shell. The symplectic structure is then defined to be:

$$
\Omega\left(\delta_{1}, \delta_{2}\right)=\int_{M} J\left(\delta_{1}, \delta_{2}\right)
$$

where $M$ is a space-like hypersurface. It follows that $d J=0$ provided the equations of motion and linearized equations of motion hold. This implies that when integrated over a closed region of spacetime bounded by $M_{+} \cup M_{-} \cup \Delta$ (where $\Delta$ is the inner boundary considered),

$$
\int_{M_{+}} J-\int_{M_{-}} J+\int_{\Delta} J=0
$$

where $M_{+}, M_{-}$are the initial and the final space-like slices, respectively. If the third term vanishes then the bulk symplectic structure is independent of choice of hypersurface. However, if it does not vanish but turns out to be exact, $\int_{\Delta} J=\int_{\Delta} d j$ then the hypersurface independent symplectic structure is given by:

$$
\Omega\left(\delta_{1}, \delta_{2}\right)=\int_{M} J-\int_{S_{\Delta}} j
$$

where $S_{\Delta}$ is the 2 -surface at the intersection of the hypersurface $M$ with the boundary $\Delta$. The quantity $j\left(\delta_{1}, \delta_{2}\right)$ is called the boundary symplectic current and symplectic structure is also independent of the choice of hypersurface.

Our strategy shall be to construct the symplectic structure for the action given in eqn. (8). Let us first look at the Lagrangian for gravity. The symplectic potential in this case is given by, $16 \pi G \Theta(\delta)=-\Sigma^{I J} \wedge \delta A_{I J}$. The symplectic current is therefore given by,

$$
J_{G}\left(\delta_{1}, \delta_{2}\right)=-\frac{1}{8 \pi G} \delta_{[1} \Sigma^{I J} \wedge \delta_{2]} A_{I J}
$$

The above expression eqn. (17), when pulled back and rescticted to the surface $\Delta$ gives

$$
\begin{aligned}
\delta_{\left[1 \Sigma^{I J} \wedge \delta_{2]} A_{I J} \triangleq\right.} & -2 \delta_{[1}{ }^{2} \epsilon \wedge \delta_{2]}\{(\epsilon+\bar{\epsilon}) n-(\alpha+\bar{\beta}) m-(\bar{\alpha}+\beta) \bar{m}\} \\
& +2 \delta_{[1}(n \wedge i m) \wedge \delta_{2]}(\bar{\rho} \bar{m})-2 \delta_{[1}(n \wedge i \bar{m}) \wedge \delta_{2]}(\rho m)
\end{aligned}
$$


It can be shown that the symplectic current pulled back on to $\Delta$ for the gravity sector is given by (see the appendix for details) ${ }^{1}$

$$
\underset{\leftarrow}{J_{G}}\left(\delta_{1}, \delta_{2}\right) \triangleq-\frac{1}{4 \pi G}\left[d\left(\delta_{[1}{ }^{2} \epsilon \delta_{2]} \log \rho\right)+\delta_{[1}{ }^{2} \epsilon \wedge \delta_{2]}\left\{\left(\frac{\Phi_{00}}{\rho}\right) n\right\}\right]
$$

The first term in the above expression is exact but not others. Therefore the phase is well defined for our boundary conditions $\sigma \triangleq 0$ provided, if either $\Phi_{00}=0$, there is no matter flux across the horizon or if $\Phi_{00} / \rho$ gets cancelled with a contribution from the matter degrees of freedom through Einstein's equation. We deal with a more general case. We show that the contribution of the scalar field is such that the symplectic current on $\Delta$ is again exact.

The symplectic current for the real scalar field is given by, $J_{M}\left(\delta_{1}, \delta_{2}\right)=2 \delta_{[1} \varphi \delta_{2]} \star d \varphi$. The symplectic current on the hypersurface $\Delta$ can be obtained as

$$
\underset{J_{M}}{\longleftarrow}\left(\delta_{1}, \delta_{2}\right)=2 \delta_{[1} \varphi \delta_{2]}(D \varphi n \wedge i m \wedge \bar{m})
$$

where $D=l^{a} \nabla_{a}$. The boundary condition on the scalar field implies $D \varphi=-2 \rho \varphi$ and hence, we get that

$$
\begin{aligned}
\underbrace{}_{M}\left(\delta_{1}, \delta_{2}\right) & =4 \delta_{[1} \varphi \delta_{2]}(-\varphi \rho n \wedge i m \wedge \bar{m}) \\
& =-d\left\{\delta_{[1} \varphi^{2} \delta_{2]}^{2} \epsilon\right\}+\delta_{[1} \frac{D \varphi D \varphi}{\rho} n \wedge \delta_{2]}^{2} \epsilon \\
& =-d\left\{\delta_{[1} \varphi^{2} \delta_{2]}^{2} \epsilon\right\}+\delta_{[1}{ }^{2} \epsilon \wedge \delta_{2]}\left(\frac{\mathbf{T}_{11}}{\rho} n\right)
\end{aligned}
$$

The combined expression is then given by:

$$
J_{M+G}\left(\delta_{1}, \delta_{2}\right) \triangleq-\frac{1}{4 \pi G}\left\{d\left(\delta_{[1}^{2} \epsilon \delta_{2]} \log \rho\right)\right\}-d\left\{\delta_{[1} \varphi^{2} \delta_{2]}{ }^{2} \epsilon\right\}
$$

It follows that the hypersurface independent symplectic structure is given by:

$$
\begin{aligned}
\Omega\left(\delta_{1}, \delta_{2}\right)=\int_{\mathcal{M}} J_{M+G}\left(\delta_{1}, \delta_{2}\right)-\int_{S_{\Delta}} j & =-\frac{1}{8 \pi G} \int_{\mathcal{M}} \delta_{[1} \Sigma^{I J} \wedge \delta_{2]} A_{I J}+2 \int_{\mathcal{M}} \delta_{[1} \varphi \delta_{2]}(\star d \varphi) \\
& +\frac{1}{4 \pi G} \int_{S_{\Delta}}\left\{\delta_{[1}^{2} \epsilon \delta_{2]} \log \rho\right\}+\int_{S_{\Delta}} \delta_{[1} \varphi^{2} \delta_{2]}^{2} \epsilon
\end{aligned}
$$

In the next section, we shall use this expression to derive the first law of mechanics for the conformal Killing horizon.

\section{Hamiltonian evolution and the first law}

Given the symplectic structure, we can proceed to study the evolution of the system. We assume that there exists a vector which gives the time evolution on the spacetime. Given this vector field, one can define a corresponding vector field on the phase- space which can be interpreted as the infinitesimal generator of time evolution in the covariant phase- space. The Hamiltonian $H_{l}$ generating the time evolution is obtained as $\delta \tilde{H}_{l}=\Omega\left(\delta, \delta_{l}\right)$, for all vector fields $\delta$ on the phase- space. Using the Einstein equations, we get that

$$
\begin{aligned}
\Omega\left(\delta, \delta_{l}\right)=-\frac{1}{16 \pi G} & \int_{S_{\Delta}}\left[\left(l . A_{I J}\right) \delta \Sigma^{I J}-\left(l . \Sigma^{I J}\right) \wedge \delta A_{I J}\right]+\int_{S_{\Delta}} \delta \varphi(l \cdot \star d \varphi) \\
& +\frac{1}{8 \pi G} \int_{S_{\Delta}}\left(\delta^{2} \epsilon \delta_{l} \log \rho-\delta_{l}{ }^{2} \epsilon \delta \log \rho\right)+\int_{S_{\Delta}} \frac{1}{2}\left(\delta \varphi^{2} \delta_{l}{ }^{2} \epsilon-\delta_{l} \varphi^{2} \delta^{2} \epsilon\right)
\end{aligned}
$$

\footnotetext{
1 The entire construction and whatever follows goes through for negative $\rho$ with the replacement $|\rho|$ in place of $\rho$ in the argument of log
} 
We now need to impose a few conditions on the fields to make a well defined Hamiltonian. These conditions are to be imposed since the action of $\delta_{l}$ on some phase- space fields is not like $£_{l}$. This is because of $\rho, \epsilon+\bar{\epsilon}$ and $\varphi$ all cannot be free data on $\Delta$. First, we note the following equalities

$$
\begin{aligned}
£_{l}\left(\frac{1}{4 \pi G} \log \rho-\frac{1}{8 \pi G} \log \varphi-\varphi^{2}\right) & =\frac{1}{4 \pi G}(2 \rho+\epsilon+\bar{\epsilon}) \\
£_{l}\left(\frac{{ }^{2} \epsilon}{\varphi}\right) & =0
\end{aligned}
$$

We assume that $\delta_{l}$ acts on $(2 \rho+\epsilon+\bar{\epsilon})$ and $\left(\frac{{ }^{2} \epsilon}{\varphi}\right)$ like $£_{l}$. This can also be argued in the following fashion. Since $\delta_{l} £_{l}(2 \rho+\epsilon+\bar{\epsilon})=0$ it immediately implies that $£_{l} \delta_{l}(2 \rho+\epsilon+\bar{\epsilon})=0$. Hence, choosing $\delta_{l}(2 \rho+\epsilon+\bar{\epsilon})=0$ at the initial cross-section implies that it remains zero throughout $\Delta$. Furthermore if we set $\delta_{l}\left(\frac{1}{4 \pi G} \log \rho-\frac{1}{8 \pi G} \log \varphi-\varphi^{2}\right)=0$ at the initial cross-section, it remains zero everywhere on $\Delta$ and so,

$$
\frac{\delta_{l} \rho}{\rho}-8 \pi G \varphi \delta_{l} \varphi-\frac{\delta_{l} \varphi}{2 \varphi}=0
$$

Another condition can be derived from the equation above

$$
\delta_{l}\left(\frac{{ }^{2} \epsilon}{\varphi}\right)=\frac{1}{\varphi} \delta_{l}^{2} \epsilon-{ }^{2} \epsilon \frac{1}{\varphi^{2}} \delta_{l} \varphi=0
$$

The variations $\delta_{l}$ satisfy the following differential equations, which can be checked to be consistent with each other:

$$
\begin{gathered}
£_{l} \delta_{l} \varphi=-2 \delta_{l} \rho \varphi-2 \rho \delta_{l} \varphi \\
£_{l} \delta_{l}{ }^{2} \epsilon=-2 \delta_{l} \rho^{2} \epsilon-2 \rho \delta_{l}{ }^{2} \epsilon
\end{gathered}
$$

Putting condition (29) in (31), we get

$$
\delta_{l} \varphi=C(\theta, \phi) \exp \left[-\int\left(16 \pi G \varphi^{2}+3\right) \rho d v\right]
$$

where $C(\theta, \phi)$, is a constant of integration. If we choose this constant $C(\theta, \phi)=0$, it immediately implies that $\delta_{l} \varphi=0=\delta_{l}^{2} \epsilon$. With the choice of $\delta_{l}$ only the bulk symplectic structure survives and one gets from eq. (26) ${ }^{2}$

$$
\delta H_{l}=-\frac{1}{8 \pi G} \int_{S_{\Delta}}(2 \rho+\epsilon+\bar{\epsilon}) \delta^{2} \epsilon+\frac{1}{8 \pi G} \int_{S_{\Delta}}{ }^{2} \epsilon(-\delta \rho-8 \pi G \delta \varphi D \varphi)+\delta E^{\infty}
$$

where we have redefined our Hamiltonian $H_{l}=\tilde{H}_{l}+\int_{S_{\Delta}} \rho^{2} \epsilon$. This redefination is possible since the definition of the Hamiltonian is ambiguous upto a total variation. Further, as expected $\Omega\left(\delta_{l}, \delta_{l}\right)=0$. Next we define, $E_{\Delta}^{l}=E^{\infty}-H_{l}$, as the horizon energy. It is clear from above that for $\rho \rightarrow 0$ (i.e in the isolated horizon limit) it matches with the definition in $[36,37]$ if asymptotics is flat and $E^{\infty}=E_{A D M}$. It therefore follows that:

$$
-\delta E_{\Delta}^{l}=-\frac{1}{8 \pi G} \int_{S_{\Delta}}(2 \rho+\epsilon+\bar{\epsilon}) \delta^{2} \epsilon-\frac{1}{8 \pi G} \int_{S_{\Delta}}\left[{ }^{2} \epsilon(\delta \rho+8 \pi G \delta \varphi D \varphi)\right] .
$$

To recover the the more familiar form of first law known for a dynamical situation, we assume there is a vector field $\tilde{\delta}$ on phase space which acts only on the fields on $\Delta$ (and not in the bulk) such that it's action on the boundary variables is to evolve the boundary fields along the affine parameter $v$ (it may be interpreted to be a time evolution, like $£)$. Now demanding that $\tilde{\delta}$ to be Hamiltonian would give an integrability condition which also ensures that $\delta_{l}$ is Hamiltonian. So one can calculate $\Omega\left(\tilde{\delta}, \delta_{l}\right):=\tilde{\delta} H_{l}$ which can be written in the following form ${ }^{3}$

$$
\dot{E}_{\Delta}^{l}=\frac{1}{8 \pi G}(2 \rho+\epsilon+\bar{\epsilon}) \dot{A}+\frac{1}{8 \pi G} \int_{S_{\Delta}}\left[{ }^{2} \epsilon(\dot{\rho}+8 \pi G \dot{\varphi} D \varphi)\right]
$$

where dots imply changes in the variables produced by the action of $\tilde{\delta}$. Note that if $\tilde{\delta}=£_{l}$, then, $\tilde{\delta} \varphi D \varphi$ gives the expression $T_{a b} l^{a} l^{b}$. Equation (36) is the form of evolution for the conformal Killing horizons. The first term in the above expression is the usual $T d S$ term while the second term is a flux term which takes into account the non-zero matter flux across $\Delta$.

\footnotetext{
${ }^{2}$ We assume that the contribution from the boundary at asymptotic infinity is a total variation $\delta E^{\infty}$.

${ }^{3}$ If the stress tensor satisfies the dominant enegy condition then $(2 \rho+\epsilon+\bar{\epsilon})$ is a constant on $\Delta$ [44].
} 


\section{DISCUSSIONS}

In this paper, we have developed the geometrical set-up for a quasi-local description of a conformal Killing horizon. Further, we have also shown that one can understand these horizons to have a zeroth law (as was also discussed in [43]) and a first law. This development of a notion of quasi-local conformal horizon should be taken in the same spirit as the development of the notion of isolated horizon from Killing horizons. A conformal Killing horizon is one which has a conformal Killing vector in the neighbourhood of the horizon. In contrast, a quasi-local conformal horizon only requires the existence of a null hypersurface generating vector which is shear free on the null hypersurface. The number of solutions of Einsteins's equation for gravity and matter that admits a conformal Killing horizon may be small (examples of such kind has been constructed by [44]). However the solutions admitting a quasi-local conformal horizon may be large. We do not comment on the nature of solutions that admits a quasi-local conformal horizon, we think that significant amount of insights may be obtained by numerical simulations and therefore falls in the regime of numerical relativity. The most useful application of these geometrical structures are in the dynamical evolution of black holes. Indeed, as matter falls in through the horizon and the black hole horizon grows, the expansion is nonzero. In such cases, it is important to understand if in this dynamical situation one can prove the existence of laws for black hole mechanics in some form.

We have taken a real scalar field as the matter field in question. The flux balance law is seen to be successfully implemented if it satisfies a condition $£_{l} \varphi \triangleq-2 \rho \varphi$. This assumption is motivated through the fact that $l^{a}$ is a conformal Killing vector on $\Delta$. Taking other matter fields will therefore be an immediate extension of our work. Further, from the onset we have ignored any space-like axial conformal Killing vector on $S_{\Delta}$. So a generalization to the rotational case seems to be another plausible extension. Since the case of an isolated horizon appears as a special case $\rho \rightarrow 0$, the consistency of our analysis can actually be checked by taking the isolated horizon limit. In fact we perform this consistency check and find that the final expressions and the first law does give back the results obtained for an isolated horizon.

We should mention at this point that our construction does not capture the most general dynamical situation, as constructed in $[34,36]$. The horizons discussed in these references are spacelike boundaries foliated by partially trapped two surfaces which may not be shear-free. Further, an integrated version of the first law has been demonstrated to exist, which captures the dynamics of growing black hole horizons in full generality. However in these constructions, which use metric variables, the existence of a well defined phase- space has not been established and consequently the first law does not follow directly from the symplectic structure. In our case we have assumed that there is no gravitational flux (shear is zero) but only matter flows across the null boundary $\Delta$. In this simplified geometry, we have demonstrated that a space of solutions of Einstein's equations exists which admit the boundary conditions of $\mathrm{CKH}$ and that a differential version of the first law of black hole mechanics can be obtained. Also, we have used the first order formalism for the construction of this symplectic structure. We do not know if one may get a well defined symplectic structure for boundary conditions discussed in [34,36]. Even if one is able to construct a phase- space, it is not possible to obtain a differential version the first law since there is no analogue of the zeroth law for such boundaries, but an integrated version of the first law is expected to hold.

Given a form of the first law, it is obvious to compare with the first law of thermodynamics. However, since the horizon is growing, it describes a non- equilibrium situation and hence may differ considerably from equilibrium thermodynamics where one studies the transition from one equilibrium state to a nearby equilibrium state. One should keep in view that thermodynamics arises out of microscopic dynamics of the underlying degrees of freedom and have universal validity (that are independent of the underlying dynamics of a particular system). For a general dynamical spacetime (when the gravitational degrees of freedom are excited), there is no time translation symmetry and hence no definition of entropy may be possible. Also in non- equilibrium cases, a system may not get enough time to relax back to the equilibrium state and hence no canonical definition of temperature exists. But, in the present scenario, though the horizon makes transition between two states which are far from equilibrium, because there exits a conformal Killing vector, this leads to a definite identification of temperature and a first law and possibly entropy. One may then enquire if dynamically growing horizons is attributed some entropy that can arise from some counting of microstates. The boundary symplectic structure has a natural interpretation of being the symplectic structure of a field theory residing on the boundary. In the case of an isolated horizon it turns out to be an $S U(2)$ or an $U(1)$ Chern-Simons theory. A quantization of the boundary theory therefore provides a microscopic description of the entropy of the isolated horizon. Since we explicitly construct the boundary symplectic structure it will be interesting to see if it does coincide with any known topological field theory. A complete answer to such questions shall have important implications for thermodynamics as well as black hole physics. 


\section{Appendix A: The Connection in terms of Newman-Penrose co-effecients}

Fix a set a internal null vectors $\left(l_{I}, n_{I}, m_{I}, \bar{m}_{I}\right)$ on $\Delta$ such that $\partial_{a}\left(l_{I}, n_{I}, m_{I}, \bar{m}_{I}\right) \triangleq 0$. Given any tetrad $e_{a}^{I}$, the null tetrad $\left(l_{a}, n_{a}, m_{a}, \bar{m}_{a}\right)$ can be expanded as $l_{a}=e_{a}^{I} l_{I}$. The expression for $\Sigma^{I J}$ can now be readily calculated and is given as.

$$
\begin{aligned}
\Sigma^{I J} & =2 l^{[I} n^{J] 2} \epsilon+2 n \wedge\left(i m l^{[I} \bar{m}^{J]}-i \bar{m} l^{[I} m^{J]}\right) \\
& -2 i l \wedge n m^{[I} \bar{m}^{J]}-2 l \wedge\left(i m n^{[I} \bar{m}^{J]}-i \bar{m} n^{[I} m^{J]}\right)
\end{aligned}
$$

This is the full expression for $\Sigma^{I J}$ where nothing has been been assumed regarding the nature of the boundary $\Delta$. If $\Delta$ is a null surface and $l_{a}$ is the null normal, we get that

$$
\sum^{I J} \triangleq 2 l^{[I} n^{J] 2} \epsilon+2 n \wedge\left(i m l^{[I} \bar{m}^{J]}-i \bar{m} l^{[I} m^{J]}\right)
$$

The covariant derivative is defined to be compatible with the tetrad i.e. $\nabla_{b} e_{a}^{I}=0$. The covariant derivatives on the null tetrads can be written in terms of the Newman-Penrose coeffecients and are given by the following,

$$
\begin{aligned}
\nabla_{a} l_{b}= & -(\epsilon+\bar{\epsilon}) n_{a} l_{b}+\bar{\kappa} n_{a} m_{b}+\kappa n_{a} \bar{m}_{b}-(\gamma+\bar{\gamma}) l_{a} l_{b}+\bar{\tau} l_{a} m_{b}+\tau l_{a} \bar{m}_{b} \\
& +\left[(\bar{\alpha}+\beta) \bar{m}_{a} l_{b}-\bar{\rho} \bar{m}_{a} m_{b}-\sigma \bar{m}_{a} \bar{m}_{b}+(\alpha+\bar{\beta}) m_{a} l_{b}-\rho m_{a} \bar{m}_{b}-\bar{\sigma} m_{a} m_{b}\right] \\
\nabla_{a} n_{b}= & (\epsilon+\bar{\epsilon}) n_{a} n_{b}-\pi n_{a} m_{b}-\bar{\pi} n_{a} \bar{m}_{b}+(\gamma+\bar{\gamma}) l_{a} n_{b}-\nu l_{a} m_{b}-\bar{\nu} l_{a} \bar{m}_{b} \\
& -\left[(\bar{\alpha}+\beta) \bar{m}_{a} n_{b}-\mu \bar{m}_{a} m_{b}-\bar{\lambda} \bar{m}_{a} \bar{m}_{b}+(\alpha+\bar{\beta}) m_{a} n_{b}-\bar{\mu} m_{a} \bar{m}_{b}-\lambda m_{a} m_{b}\right] \\
\nabla_{a} m_{b}= & -\bar{\pi} n_{a} l_{b}+\kappa n_{a} n_{b}-(\epsilon-\bar{\epsilon}) n_{a} m_{b}-\bar{\nu} l_{a} l_{b}+\tau l_{a} n_{b}-(\gamma-\bar{\gamma}) l_{a} m_{b} \\
& +\left[\bar{\lambda} \bar{m}_{a} l_{b}-\sigma \bar{m}_{a} n_{b}+(\beta-\bar{\alpha}) \bar{m}_{a} m_{b}+\bar{\mu} m_{a} l_{b}-\rho m_{a} n_{b}+(\alpha-\bar{\beta}) m_{a} m_{b}\right]
\end{aligned}
$$

Next, once we have fixed a set of null internal vectors on $\Delta$, the connection can be expanded in terms of these Newman- Penrose coefficients. Note that $\nabla_{a} l_{I}=\partial_{a} l_{I}+A_{a I}^{J} l_{J}$. Therefore on $\Delta$, we have $e_{I}^{b} \nabla_{a} l_{b} \triangleq A_{a I}{ }^{J} l_{J}$ and hence

$$
\begin{aligned}
A_{a I}^{(l) J} l_{J} \triangleq & -(\epsilon+\bar{\epsilon}) n_{a} l_{I}+\bar{\kappa} n_{a} m_{I}+\kappa n_{a} \bar{m}_{I}-(\gamma+\bar{\gamma}) l_{a} l_{I}+\bar{\tau} l_{a} m_{I}+\tau l_{a} \bar{m}_{I} \\
& +\left[(\bar{\alpha}+\beta) \bar{m}_{a} l_{I}-\bar{\rho} \bar{m}_{a} m_{I}-\sigma \bar{m}_{a} \bar{m}_{I}+(\alpha+\bar{\beta}) m_{a} l_{I}-\rho m_{a} \bar{m}_{I}-\bar{\sigma} m_{a} m_{I}\right] \\
A_{a I J}^{(l) \triangleq} & {\left[(\epsilon+\bar{\epsilon}) n_{a}+(\gamma+\bar{\gamma}) l_{a}-(\bar{\alpha}+\beta) \bar{m}_{a}-(\alpha+\bar{\beta}) m_{a}\right] 2 l_{[I} n_{J]} } \\
& +\left[-\bar{\kappa} n_{a}-\bar{\tau} l_{a}+\bar{\rho} \bar{m}_{a}+\bar{\sigma} m_{a}\right] 2 m_{[I} n_{J]}+\left[-\kappa n_{a}-\tau l_{a}+\rho m_{a}+\sigma \bar{m}_{a}\right] 2 \bar{m}_{[I} n_{J]}
\end{aligned}
$$

where the subscript $l$ in $A^{(l)}$ indicates that the only the vector field $l^{a}$ has been used to evaluate the connection. Similarly, we can proceed for other vector fields $n^{a}, m^{a}$ and $\bar{m}^{a}$. The resulting connections are given as follows

$$
\begin{aligned}
A_{a I J}^{(n)} \triangleq & {\left[-(\epsilon+\bar{\epsilon}) n_{a}-(\gamma+\bar{\gamma}) l_{a}+(\bar{\alpha}+\beta) \bar{m}_{a}+(\alpha+\bar{\beta}) m_{a}\right] 2 n_{[I} l_{J]} } \\
& +\left(\pi n_{a}+\nu l_{a}-\mu \bar{m}_{a}-\lambda m_{a}\right) 2 m_{[I} l_{J]}+\left(\bar{\pi} n_{a}+\bar{\nu} l_{a}-\bar{\mu} m_{a}-\bar{\lambda} \bar{m}_{a}\right) 2 \bar{m}_{[I} l_{J]} \\
A_{a I J}^{(m) \triangleq} & \left(-\bar{\pi} n_{a}-\bar{\nu} l_{a}+\bar{\lambda} \bar{m}_{a}+\bar{\mu} m_{a}\right) 2 l_{[I} \bar{m}_{J]}+\left(\kappa n_{a}+\tau l_{a}-\sigma \bar{m}_{a}-\rho m_{a}\right) 2 n_{[I} \bar{m}_{J]} \\
& +\left[-(\epsilon-\bar{\epsilon}) n_{a}-(\gamma-\bar{\gamma}) l_{a}+(\alpha-\bar{\beta}) m_{a}+(\beta-\bar{\alpha}) \bar{m}_{a}\right] 2 m_{[I} \bar{m}_{J]}
\end{aligned}
$$


The full connection is then given by:

$$
\begin{aligned}
A_{a I J} \triangleq & 2\left[(\epsilon+\bar{\epsilon}) n_{a}+(\gamma+\bar{\gamma}) l_{a}-(\bar{\alpha}+\beta) \bar{m}_{a}-(\alpha+\bar{\beta}) m_{a}\right] l_{[I} n_{J]} \\
& +2\left[-\bar{\kappa} n_{a}-\bar{\tau} l_{a}+\bar{\rho} \bar{m}_{a}+\bar{\sigma} m_{a}\right] m_{[I} n_{J]}+2\left[-\kappa n_{a}-\tau l_{a}+\rho m_{a}+\sigma \bar{m}_{a}\right] \bar{m}_{[I} n_{J]} \\
& +2\left[\pi n_{a}+\nu l_{a}-\mu \bar{m}_{a}-\lambda m_{a}\right] m_{[I} l_{J]}+2\left[\bar{\pi} n_{a}+\bar{\nu} l_{a}-\bar{\mu} m_{a}-\bar{\lambda} \bar{m}_{a}\right] \bar{m}_{[I} l_{J]} \\
& +2\left[-(\epsilon-\bar{\epsilon}) n_{a}-(\gamma-\bar{\gamma}) l_{a}+(\alpha-\bar{\beta}) m_{a}+(\beta-\bar{\alpha}) \bar{m}_{a}\right] m_{[I} \bar{m}_{J]}
\end{aligned}
$$

Note that as in in the case of $\Sigma_{I J}$ no boundary condition has been assumed in the above expression. In the main part of the paper this expression for the connection eqn (10) shall be used but with the boundary conditions.

Further, we would be requiring the exterior derivatives on the null tetrads. We therefore give the expressions here.

$$
\begin{aligned}
& d n=\nabla_{a} n_{b} d x^{a} \wedge d x^{b}=-\pi n \wedge m-\bar{\pi} n \wedge \bar{m}+(\gamma+\bar{\gamma}) l \wedge n-\nu l \wedge m-\bar{\nu} l \wedge \bar{m} \\
& -[(\bar{\alpha}+\beta) \bar{m} \wedge n-\mu \bar{m} \wedge m+(\alpha+\bar{\beta}) m \wedge n-\bar{\mu} m \wedge \bar{m}] \\
& d l=\nabla_{a} l_{b} d x^{a} \wedge d x^{b}=-(\epsilon+\bar{\epsilon}) n \wedge l+\bar{\kappa} n \wedge m+\kappa n \wedge \bar{m}+\bar{\tau} l \wedge m+\tau l \wedge \bar{m} \\
& +[(\bar{\alpha}+\beta) \bar{m} \wedge l-\bar{\rho} \bar{m} \wedge m+(\alpha+\bar{\beta}) m \wedge l-\rho m \wedge \bar{m}] \\
& d m=\nabla_{a} m_{b} d x^{a} \wedge d x^{b}=-\bar{\pi} n \wedge l-(\epsilon-\bar{\epsilon}) n \wedge m+\tau l \wedge n-(\gamma-\bar{\gamma}) l \wedge m \\
& +[\bar{\lambda} \bar{m} \wedge l-\sigma \bar{m} \wedge n+(\beta-\bar{\alpha}) \bar{m} \wedge m+\bar{\mu} m \wedge l-\rho m \wedge n]
\end{aligned}
$$

From the above expressions, it follows that for the area two- form which is given by ${ }^{2} \epsilon=i m \wedge \bar{m}$, we get that $d^{2} \epsilon=2 \rho n \wedge{ }^{2} \epsilon$ and $£_{l}^{2} \epsilon=-2 \rho^{2} \epsilon$.

\section{Appendix B: Variation of the action}

Since the boundary symplectic structure turned out to be exact, it is at once evident that the variation of the action should be well-defined with the the boundary conditions considered. However one may need to add an additional boundary term in order to it. As has been pointed out that such terms won't affect the symplectic structure though. Therefore for completeness we consider the variation of the action and find out the necessary boundary term needed to make the variation well defined. We consider the action for gravity and a scalar field without any boundary terms a priori. The expression for $\Theta$ on $\Delta$ is calculated imposing the boundary conditions and the required boundary term can be obtained. We have

$$
L_{M+G}=-\frac{1}{16 \pi G}\left(\Sigma^{I J} \wedge F_{I J}\right)-\frac{1}{2} d \varphi \wedge \star d \varphi
$$

It follows that

$$
d \Theta(\delta)=-\frac{1}{16 \pi G} d\left(\Sigma^{I J} \wedge \delta A_{I J}\right)-d(\delta \varphi \star d \varphi)
$$

Consider the gravity terms first ${ }^{4}$

$$
\begin{aligned}
\sum^{\Sigma^{I J} \wedge \delta A_{I J}} & \triangleq-2^{2} \epsilon \wedge \delta[(\epsilon+\bar{\epsilon}) n]+2(n \wedge i m) \wedge \delta(\rho \bar{m})-2(n \wedge i \bar{m}) \wedge \delta(\rho m) \\
& =-2^{2} \epsilon \wedge \delta\left[\left(\frac{D \rho}{\rho}-\rho-\frac{\Phi_{00}}{\rho}\right) n\right]+2(n \wedge i m) \wedge \delta(\rho \bar{m})-2(n \wedge i \bar{m}) \wedge \delta(\rho m) \\
& =d\left[2^{2} \epsilon \delta(\log \rho)\right]-4 n \wedge^{2} \epsilon \delta \rho+2^{2} \epsilon \wedge \delta\left[\left(\rho+\frac{\Phi_{00}}{\rho}\right) n\right]+4 n \wedge^{2} \epsilon \delta \rho+2 \rho n \wedge \delta^{2} \epsilon \\
& =d\left[2^{2} \epsilon \delta(\log \rho)\right]+2^{2} \epsilon \wedge \delta\left[\left(\frac{\mathbf{R}_{11}}{2 \rho}\right) n\right]+\delta\left(2 \rho n \wedge^{2} \epsilon\right)
\end{aligned}
$$

\footnotetext{
${ }^{4}$ In our case it might not be possible to define a unique covariant derivative on $\Delta$. However, since in the the calculations $l^{a} \nabla_{a}$ acts only on functions, the amibiguity do not play a role.
} 
The matter term gives

$$
\begin{aligned}
(\delta \varphi \star d \varphi) & =-d\left(\frac{1}{2} \delta \varphi^{2}{ }^{2} \epsilon\right)+\delta(\varphi d \varphi) \wedge{ }^{2} \epsilon \\
& =-d\left(\frac{1}{2} \delta \varphi^{2}{ }^{2} \epsilon\right)-\frac{1}{2} \delta\left(\frac{\mathbf{T}_{11}}{\rho} n\right) \wedge{ }^{2} \epsilon
\end{aligned}
$$

Adding everything up, one finds that,

$$
d \Theta(\delta)=-\frac{1}{16 \pi G} d\left(\Sigma^{I J} \wedge \delta A_{I J}\right)-d(\delta \varphi \star d \varphi)=-\frac{1}{8 \pi G} d \delta\left(\rho n \wedge{ }^{2} \epsilon\right)
$$

So one needs to add $\frac{1}{8 \pi G} \int_{\Delta}\left(\rho n \wedge^{2} \epsilon\right)$ to the action to make the variation well-defined.

\section{Appendix C: Boundary Symplectic Structure for Gravity}

The symplectic current in first order gravity is therefore given by,

$$
J_{G}\left(\delta_{1}, \delta_{2}\right)=-\frac{1}{8 \pi G} \delta_{[1} \Sigma^{I J} \wedge \delta_{2]} A_{I J}
$$

We need to pull back the above expression on to the boundary and check if it is exact.

$$
\begin{aligned}
\delta_{[1} \Sigma^{I J} \wedge \delta_{2]} A_{I J} \triangleq & -2 \delta_{[1}{ }^{2} \epsilon \wedge \delta_{2]}((\epsilon+\bar{\epsilon}) n-(\alpha+\bar{\beta}) m-(\bar{\alpha}+\beta) \bar{m}) \\
& +2 \delta_{[1}(n \wedge i m) \wedge \delta_{2]}(\bar{\rho} \bar{m})-2 \delta_{[1}(n \wedge i \bar{m}) \wedge \delta_{2]}(\rho m)
\end{aligned}
$$

We consider the first term in the above expression. By using the Ricci identity in terms of Newman-Penrose co-effecients

$$
D \rho=\rho^{2}+\rho(\epsilon+\bar{\epsilon})+\Phi_{00}
$$

we find that the first term can be written in the following form,

$$
\begin{aligned}
&-2 \delta_{[1}{ }^{2} \epsilon \wedge \delta_{2]}((\epsilon+\bar{\epsilon}) n)=-2 \delta_{[1}{ }^{2} \epsilon \wedge \delta_{2]}\left(\left(\frac{D \rho}{\rho}-\frac{\rho^{2}}{\rho}-\frac{\Phi_{00}}{\rho}\right) n\right) \\
&=d\left(2 \delta_{[1}{ }^{2} \epsilon \delta_{2]} \log \rho\right)-\left(2 \delta_{[1} d^{2} \epsilon \wedge \delta_{2]} \log \rho\right) \\
&+2 \delta_{[1}{ }^{2} \epsilon \wedge \delta_{2]}\left(\left(\frac{\rho^{2}}{\rho}+\frac{\Phi_{00}}{\rho}\right) n\right)
\end{aligned}
$$

Since the first term in the above expression is already exact, we leave it for the the moment and check if there is any simplication of the other terms when combined with the rest of the third and forth term in the symplectic current.

$$
\begin{aligned}
& -2 \delta_{[1} d^{2} \epsilon \wedge \delta_{2]} \log \rho=-4 \delta_{[1} i \rho n \wedge m \wedge \bar{m} \delta_{2]} \log \rho \\
& =-2 \delta_{[1}(n \wedge i m) \wedge \bar{m} \delta_{2]} \rho-2(n \wedge i m) \wedge \delta_{[1} \bar{m} \delta_{2]} \rho \\
& +2 \delta_{[1}(n \wedge i \bar{m}) \wedge m \delta_{2]} \rho+2(n \wedge i \bar{m}) \wedge \delta_{[1} m \delta_{2]} \rho
\end{aligned}
$$

The third and the fourth term in the symplectic current gives:

$$
\begin{aligned}
& 2 \delta_{[1}(n \wedge i m) \wedge \delta_{2]}(\rho \bar{m})-2 \delta_{[1}(n \wedge i \bar{m}) \wedge \delta_{2]}(\rho m) \\
&=2 \delta_{[1}(n \wedge i m) \wedge \bar{m} \delta_{2]}(\rho)+2 \rho \delta_{[1}(n \wedge i m) \wedge \delta_{2]} \bar{m} \\
&-2 \delta_{[1}(n \wedge i \bar{m}) \wedge m \delta_{2]}(\rho)-2 \rho \delta_{[1}(n \wedge i \bar{m}) \wedge \delta_{2]} m
\end{aligned}
$$

Adding the above two equations and then simplifying gives: 


$$
\begin{aligned}
-2 \delta_{[1} d^{2} \epsilon \wedge \delta_{2]} \log \rho & +2 \delta_{[1}(n \wedge i m) \wedge \delta_{2]}(\rho \bar{m})-2 \delta_{[1}(n \wedge i \bar{m}) \wedge \delta_{2]}(\rho m) \\
& =-2 n \wedge \delta_{[1}{ }^{2} \epsilon \delta_{2]} \rho+2 \rho \delta_{[1}(n) \wedge \delta_{2]}{ }^{2} \epsilon \\
& =-2 \delta_{[1}{ }^{2} \epsilon \wedge \delta_{2]}(\rho n)
\end{aligned}
$$

So the boundary term becomes

$$
d\left(2 \delta_{[1}^{2} \epsilon \delta_{2]} \log \rho\right)+2 \delta_{[1}^{2} \epsilon \wedge \delta_{2]}\left(\frac{\Phi_{00}}{\rho} n\right)
$$

\section{Appendix D: Bulk Symplectic structure}

For any vector field $\xi$ generating diffeomorphisms, the corresponding phase space variation $\delta_{\xi}$ acts in the bulk like $£_{\xi}$. It can then be shown that

$$
J_{G}\left(\delta, \delta_{\xi}\right)=-\frac{1}{16 \pi G}\left[\left(\xi \cdot A_{I J}\right) \delta \Sigma^{I J}-\left(\xi \cdot \Sigma^{I J}\right) \wedge \delta A_{I J}\right]+(\text { Equations of motion }) \delta e^{I}
$$

Similarly for the matter fields, we get that

$$
J_{M}\left(\delta, \delta_{\xi}\right)=d[\delta \varphi(\xi . \star d \varphi)]-[\delta d \varphi(\xi . \star d \varphi)]-\xi . d \varphi \delta(\star d \varphi)
$$

The second and the third term in the last expression enters Einstein's equation. Therefore the full bulk symplectic structure is,

$$
\int_{M} J\left(\delta, \delta_{\xi}\right)=-\frac{1}{16 \pi G} \int_{\partial M}\left[\left(\xi . A_{I J}\right) \delta \Sigma^{I J}-\left(\xi . \Sigma^{I J}\right) \wedge \delta A_{I J}\right]+\int_{\partial M} \delta \varphi(\xi . \star d \varphi)
$$

\section{Acknowedgements}

The authors acknowledge the discussions with Amit Ghosh. The authors also thank the anonymous referee for suggestions that made the presentation better. AC is partially supported through the UGC- BSR start-up grant vide their letter no. F.20-1(30)/2013(BSR)/3082. AG is supported by Department of Atomic-Energy, Govt. Of India.

[1] S. W. Hawking and G. F. R. Ellis, "The Large scale structure of space-time," Cambridge University Press, Cambridge, 1973

[2] R. M. Wald, "General Relativity," Chicago, USA: Chicago Univ. Pr. ( 1984) 491p

[3] S. W. Hawking, Commun. Math. Phys. 25, 152 (1972).

[4] J. M. Bardeen, B. Carter and S. W. Hawking, "The Four laws of black hole mechanics," Commun. Math. Phys. 31, 161 (1973).

[5] S. W. Hawking, "Particle Creation by Black Holes," Commun. Math. Phys. 43, 199 (1975) [Erratum-ibid. 46, 206 (1976)].

[6] J. D. Bekenstein, "Black holes and entropy," Phys. Rev. D 7, 2333 (1973).

[7] J. D. Bekenstein, "Generalized second law of thermodynamics in black hole physics," Phys. Rev. D 9, 3292 (1974).

[8] R. M. Wald, "Quantum field theory in curved space-time and black hole thermodynamics," Chicago, USA: Univ. Pr. (1994) $205 \mathrm{p}$

[9] R. M. Wald, "Black hole entropy is the Noether charge," Phys. Rev. D 48, 3427 (1993) [gr-qc/9307038].

[10] V. Iyer and R. M. Wald, "Some properties of Noether charge and a proposal for dynamical black hole entropy," Phys. Rev. D 50, 846 (1994) [gr-qc/9403028].

[11] T. Jacobson, G. Kang and R. C. Myers, "On black hole entropy," Phys. Rev. D 49, 6587 (1994) [gr-qc/9312023].

[12] D. Youm, "Black holes and solitons in string theory," Phys. Rept. 316, 1 (1999) [hep-th/9710046].

[13] S. Carlip, "Entropy from conformal field theory at Killing horizons," Class. Quant. Grav. 16, 3327 (1999) [gr-qc/9906126].

[14] O. Dreyer, A. Ghosh and A. Ghosh, "Entropy from near-horizon geometries of Killing horizons," Phys. Rev. D 89, 024035 (2014) [arXiv:1306.5063 [gr-qc]]. 
[15] A. Ghosh, "Note on Kerr/CFT correspondence in a first order formalism," Phys. Rev. D 89, 124035 (2014) [arXiv:1404.5260 [gr-qc]].

[16] A. Ashtekar, C. Beetle and S. Fairhurst, "Isolated horizons: A Generalization of black hole mechanics," Class. Quant. Grav. 16, L1 (1999) [gr-qc/9812065].

[17] A. Ashtekar, C. Beetle, O. Dreyer, S. Fairhurst, B. Krishnan, J. Lewandowski and J. Wisniewski, "Isolated horizons and their applications," Phys. Rev. Lett. 85 (2000) 3564 [gr-qc/0006006].

[18] A. Ashtekar, S. Fairhurst and B. Krishnan, "Isolated horizons: Hamiltonian evolution and the first law," Phys. Rev. D 62 (2000) 104025 [gr-qc/0005083].

[19] A. Ashtekar, C. Beetle and J. Lewandowski, "Mechanics of rotating isolated horizons," Phys. Rev. D 64, 044016 (2001) [gr-qc/0103026].

[20] A. Ashtekar, C. Beetle and J. Lewandowski, "Geometry of generic isolated horizons," Class. Quant. Grav. 19, 1195 (2002) [gr-qc/0111067].

[21] A. Chatterjee and A. Ghosh, "Generic weak isolated horizons," Class. Quant. Grav. 23, 7521 (2006) [gr-qc/0603023].

[22] A. Chatterjee and A. Ghosh, "Laws of Black Hole Mechanics from Holst Action," Phys. Rev. D 80, 064036 (2009) [arXiv:0812.2121 [gr-qc]].

[23] L. Smolin, "Linking topological quantum field theory and nonperturbative quantum gravity," J. Math. Phys. 36, 6417 (1995) [gr-qc/9505028].

[24] K. V. Krasnov, "Counting surface states in the loop quantum gravity," Phys. Rev. D 55, 3505 (1997) [gr-qc/9603025].

[25] C. Rovelli, "Black hole entropy from loop quantum gravity," Phys. Rev. Lett. 77, 3288 (1996) [gr-qc/9603063].

[26] A. Ashtekar, J. Baez, A. Corichi and K. Krasnov, "Quantum geometry and black hole entropy," Phys. Rev. Lett. 80, 904 (1998) [gr-qc/9710007].

[27] A. Ashtekar, A. Corichi and K. Krasnov, "Isolated horizons: The Classical phase space," Adv. Theor. Math. Phys. 3, 419 (1999) [gr-qc/9905089].

[28] A. Ghosh and P. Mitra, "Counting black hole microscopic states in loop quantum gravity," Phys. Rev. D 74, 064026 (2006) [hep-th/0605125].

[29] A. Ghosh and P. Mitra, "Fine-grained state counting for black holes in loop quantum gravity," Phys. Rev. Lett. 102, 141302 (2009) [arXiv:0809.4170 [gr-qc]].

[30] A. Ghosh and A. Perez, "Black hole entropy and isolated horizons thermodynamics," Phys. Rev. Lett. 107, 241301 (2011) [Erratum-ibid. 108, 169901 (2012)] [arXiv:1107.1320 [gr-qc]].

[31] A. Ghosh, K. Noui and A. Perez, "Statistics, holography, and black hole entropy in loop quantum gravity," arXiv:1309.4563 [gr-qc].

[32] A. Corichi, I. Rubalcava and T. Vukasinac, "First order gravity: Actions, topological terms and boundaries," arXiv:1312.7828 [gr-qc].

[33] A. Corichi, I. Rubalcava-Garca and T. Vukainac, "Hamiltonian and Noether charges in first order gravity," Gen. Rel. Grav. 46, $1813(2014)$.

[34] S. A. Hayward, "General laws of black hole dynamics," Phys. Rev. D 49, 6467 (1994).

[35] S. A. Hayward, "Spin coefficient form of the new laws of black hole dynamics," Class. Quant. Grav. 11, 3025 (1994) [gr-qc/9406033].

[36] A. Ashtekar and B. Krishnan, "Dynamical horizons: Energy, angular momentum, fluxes and balance laws," Phys. Rev. Lett. 89, 261101 (2002) [gr-qc/0207080].

[37] A. Ashtekar and B. Krishnan, "Dynamical horizons and their properties," Phys. Rev. D 68, 104030 (2003) [gr-qc/0308033].

[38] A. Ashtekar and B. Krishnan, "Isolated and dynamical horizons and their applications," Living Rev. Rel. 7, 10 (2004) [gr-qc/0407042].

[39] I. Booth and S. Fairhurst, "The First law for slowly evolving horizons," Phys. Rev. Lett. 92, 011102 (2004) [gr-qc/0307087].

[40] I. Booth and S. Fairhurst, "Isolated, slowly evolving, and dynamical trapping horizons: Geometry and mechanics from surface deformations," Phys. Rev. D 75, 084019 (2007) [gr-qc/0610032].

[41] I. Booth and S. Fairhurst, "Extremality conditions for isolated and dynamical horizons," Phys. Rev. D 77, 084005 (2008) [arXiv:0708.2209 [gr-qc]].

[42] C. C. Dyer and E. Honig, J. Math. Phys. 20, 409 (1979)

[43] J. Sultana and C. C. Dyer, J. Math. Phys. 45, 4764 (2004)

[44] J. Sultana and C. C. Dyer, "Cosmological black holes: A black hole in the Einstein-de Sitter universe," Gen. Rel. Grav. 37, 1347 (2005).

[45] T. Jacobson and G. Kang, "Conformal invariance of black hole temperature," Class. Quant. Grav. 10, L201 (1993) [grqc/9307002].

[46] A. B. Nielsen and J. T. Firouzjaee, "Conformally rescaled spacetimes and Hawking radiation," Gen. Rel. Grav. 45, 1815 (2013) [arXiv:1207.0064 [gr-qc]].

[47] S. Chandrasekhar, "The mathematical theory of black holes," Oxford, UK: Clarendon (1985) 646 P.

[48] G. J. Galloway and R. Schoen, "A Generalization of Hawking's black hole topology theorem to higher dimensions," Commun. Math. Phys. 266, 571 (2006) [gr-qc/0509107]. 